\title{
Tratamiento de una lesión endoperiodontal tipo III (combinada o verdadera): reporte de un caso
}

\author{
Treatment of type III periodontal-endodontic lesion (combined or true): \\ a case report
}

Alcota $\mathrm{M}^{1}$, Mondragón $\mathrm{R}^{2}$, Zepeda $\mathrm{C}^{3}$

\begin{abstract}
RESUMEN
Se presenta un caso clínico de una lesión endoperiodontal tipo III (combinada o verdadera) en un paciente de sexo femenino de 41 años de edad sin antecedentes sistémicos. La paciente fue derivada del Curso de Especialización en Periodoncia de la Escuela de Graduados de la Universidad de Chile debido a una lesión periapical en la pieza 3.6. El pronóstico en este tipo de lesiones es dudoso, ya que es necesario que se efectúe el tratamiento endodóntico así como el periodontal, y el resultado recae más en el tratamiento periodontal. La pieza fue tratada endodónticamente dejando medicamento intraconducto a base de Clorhexidina al $2 \%$ en gel por 7 días. Una vez obturado el diente se citó a la paciente 3 meses después para un control radiográfico. Actualmente el diente está totalmente asintomático, sin movilidad y con señales de reparación.
\end{abstract}

Rev. Clin. Periodoncia Implantol. Rehabil. Oral Vol. 4(1); 26-28, 2011.

Palabras clave: Lesión endoperiodontal, diagnóstico, pronóstico.

\section{ABSTRACT}

We report a case of a type III periodontal-endodontic lesion (combined or true) in a 41-year-old female patient without systemic history. The patient was transferred from the Specialization Course in Periodontology at the Graduate School of the University of Chile due to a periapical lesion in the tooth 3.6. The prognosis for this type of lesion is uncertain, since it is necessary to perform endodontic and periodontal treatment, and the result depends more on the periodontal treatment. The piece was treated endodontically leaving intracanal medication based on $2 \%$ Chlorhexidine gel for 7 days. Once the tooth obturated, we gave the patient an appointment 3 months later for a control radiography. Currently, the tooth is completely asymptomatic, without mobility and with signs of repair.

Rev. Clin. Periodoncia Implantol. Rehabil. Oral Vol. 4(1); 26-28, 2011.

Key words: Periodontal-endodontic lesions, diagnosis, prognosis.

\section{INTRODUCCIÓN}

La relación dinámica entre la pulpa y el periodonto debe considerarse como una unidad biológica, la cual requiere para su función normal un buen estado de salud, tanto de sus tejidos dentales como de los tejidos periodontales o de soporte ${ }^{(1)}$. En las lesiones endoperiodontales juega un rol fundamental las diferentes interconexiones anatómicas y vasculares entre la pulpa y el periodonto. Desde el desarrollo del germen dental se forman estructuras como los túbulos dentinarios y conductos principales, secundarios, laterales, entre otros, los cuales son capaces de comunicar las estructuras endodónticas y periodontales y de esta manera permitir un intercambio de sustancias entre ambas, razón por la cual es común que una enfermedad pulpar afecte las estructuras periodontales y viceversa ${ }^{(2)}$. En la raíz, los túbulos dentinarios se extienden desde la pulpa a la unión amelocementaria y se pueden exponer por defectos de desarrollo, enfermedad o procedimientos periodontales. Los conductos laterales y accesorios pueden presentarse a lo largo de toda la raíz, siendo más frecuente a nivel apical. En la furca, estos conductos accesorios son una vía directa de comunicación entre pulpa y periodonto, a través de tejido conectivo y vasos contenidos en los conductos. Por último, el foramen apical es la ruta principal de comunicación; las bacterias y productos inflamatorios existentes en los conductos radiculares pueden extenderse a los tejidos periapicales, causando una respuesta inflamatoria local que puede ir acompañada de reabsorción ósea y radicular. A su vez, el foramen apical es una puerta de entrada a la pulpa para las bacterias y productos inflamatorios desde sacos periodontales profundos que se extienden hasta éste ${ }^{(1-5)}$.
Tantolaenfermedad periodontalcomolaendodónticapresentan una naturaleza infecciosa y muchas de las especies encontradas en los conductos radiculares pueden estar también presentes en los sacos periodontales ${ }^{(2-6)}$. Cualquiera de estas enfermedades puede imitar a la otra tanto clínica como radiográficamente, por lo tanto un diagnóstico preciso de los factores etiológicos involucrados es necesario para un correcto tratamiento ${ }^{(3)}$.

Cuando se presenta una lesión endoperiodontal se debe buscar el origen de dicha infección, realizando un diagnóstico diferencial entre enfermedad endodóntica, periodontal o combinada ${ }^{(2,5)}$.

Clínicamente las lesiones endoperiodontales se han clasificado de acuerdo a su etiología, sugiriéndose varias clasificaciones para dividir los casos que pueden requerir terapias simples o combinadas. De esta manera una de las clasificaciones más utilizadas en los últimos tiempos por su simpleza y fácil aplicación fue la descrita por Guldener \& Langeland en $1982^{(7):}$

Tipo I: Lesión esencialmente endodóntica. Origen endodóntico, exacerbación aguda de lesión apical crónica que drena desde el ligamento al surco y simula un absceso periodontal, con aumento de volumen marginal.

Tipo II: Lesión esencialmente periodontal. Origen periodontal, enfermedad periodontal que destruye tejidos de soporte y afecta la pulpa.

Tipo III: Lesión endoperiodontal combinada o verdadera. En las cuales ambos procesos de patología endodóntica y periodontal se producen conjuntamente.

1. Cirujano Dentista. Magíster en Educación en Ciencias de la Salud. Especialista en Endodoncia. Departamento de Odontología Conservadora, Facultad de Odontología, Universidad de Chile. Chile.

2. Cirujano Dentista. Especialista en Endodoncia, Universidad de Chile. Ejercicio Particular. Chile.

3. Cirujano Dentista. Especialista en Endodoncia, Universidad de Chile. CDT Eloísa Díaz, Servicio Metropolitano de Salud Norte. Chile. 
A continuación se describe el caso y el tratamiento de una pieza 3.6 con una lesión endoperiodontal verdadera.

\section{CASO CLÍNICO}

Paciente de sexo femenino, de 41 años de edad, actualmente asintomática, acude a la Clínica de Endodoncia de la Escuela de Graduados de la Universidad de Chile derivada de la Clínica de Periodoncia de la misma universidad, para realizar el tratamiento endodóntico de la pieza 3.6. La paciente relata haber tenido dolor en el diente varios meses atrás, nunca fue atendida.

La paciente presentaba un estado general de salud bueno, sin alergias conocidas ni enfermedades, informando que padeció de hipertensión durante el embarazo y nunca volvió a tener ningún inconveniente. No se encontraba bajo ningún tratamiento médico ni farmacológico.

Clínicamente, se observó en el diente una restauración penetrante defectuosa y respuesta negativa a los test de sensibilidad. Además, presentaba un compromiso de furca grado I por vestibular y lingual, con encía marginal inflamada, sangrado al sondaje periodontal en los sitios evaluados y sacos periodontales que por la cara vestibular alcanzaban una profundidad de $6 \mathrm{~mm}$ en la medición mesiovestibular con presencia de exudado a la presión digital por vestibular y movilidad grado 2 .

Al examen radiográfico se detectó una restauración profunda penetrante y una zona radiolúcida que abarcaba desde el periápice hasta aproximadamente el tercio medio de la raíz mesiovestibular (tamaño 8 $\mathrm{mm}$ vertical por $3 \mathrm{~mm}$ horizontal). Se observaba además pérdida ósea marginal horizontal y vertical por distal del diente, y una cámara y conductos radiculares normales (Figura 1).

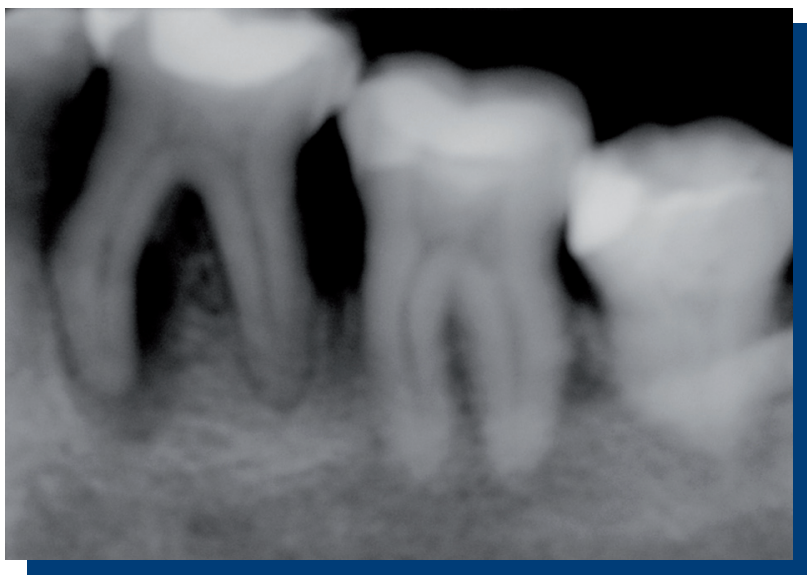

Figura 1. Radiografía inicial

Al realizar la cavidad de acceso fue encontrado un diente desvitalizado, corroborando las respuestas a los test de sensibilidad, además se detectó la presencia de supuración en el conducto mesiovestibular. Se determinó la longitud de trabajo utilizando ambos métodos radiográfico y electrónico (localizador apical) MV: 19 mm, ML: 19 $\mathrm{mm}$ y D: $19 \mathrm{~mm}$. La técnica de instrumentación a utilizar fue coronoapical mecanizada, utilizando limas Race en los conductos mesiales hasta llegar al número $25 / 0.04 \%$ terminando con una lima \# 30 manual K (Maillefer) hasta la longitud de trabajo. En el conducto distal se utilizó limas Race llegando hasta el número 30/0.04\% y una lima \# 35 manual K (Maillefer) hasta longitud de trabajo. Se irrigó abundantemente con $\mathrm{NaOCl}$ al $5.25 \%$ entre el uso de cada lima. Se dejó clorhexidina en gel al $2 \%$ dentro del conducto como medicamento, se realizó doble sellado cameral (fermin y vidrioionómero) y se citó al paciente en una semana (Figura 2).

En una segunda sesión se limpió el conducto con suero fisiológico, se repasó la instrumentación con la lima maestra respectiva en cada uno de los conductos hasta la longitud de trabajo establecida, complementando con abundante irrigación con $\mathrm{NaOCl}$ al $5.25 \%$. Se secaron los canales y se realizó la obturación de éstos, los canales mesiales fueron obturados con cono principal \# 30 y el canal distal con cono principal \# 35, agregando conos accesorios R7 y R8, utilizando como sellador el cemento Top Seal. Se procedió a realizar la radiografía de control de obturación (Figura 3).

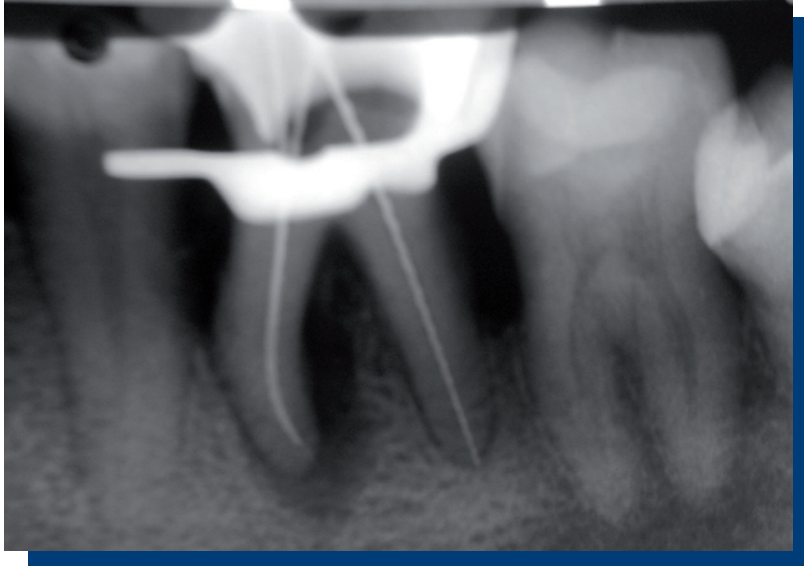

Figura 2. Control de longitud.

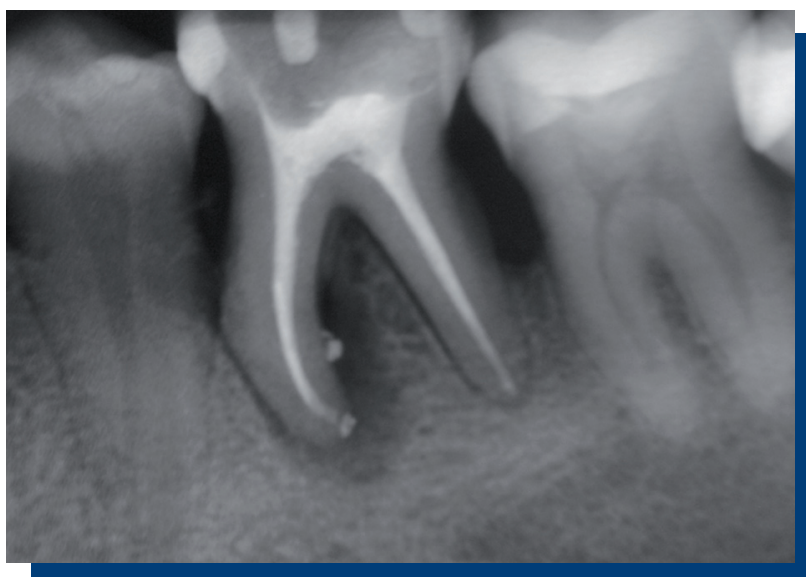

Figura 3. Control de obturación.

Se citó a la paciente una semana despúes para control clinico y dar el alta. Se derivó nuevamente al periodoncista para que le diese continuidad al tratamiento periodontal. Se citó al paciente para tres meses más para un control clínico radiográfico.

Al control de los tres meses, clínicamente la paciente se encuentra asintomática y la pieza dentaria ya no presenta movilidad, ni sacos periodontales, ni sangramiento al sondaje periodontal. Radiográficamente se puede observar que la lesión remitió casi en su totalidad, siendo esto una señal de reparación (Figura 4).

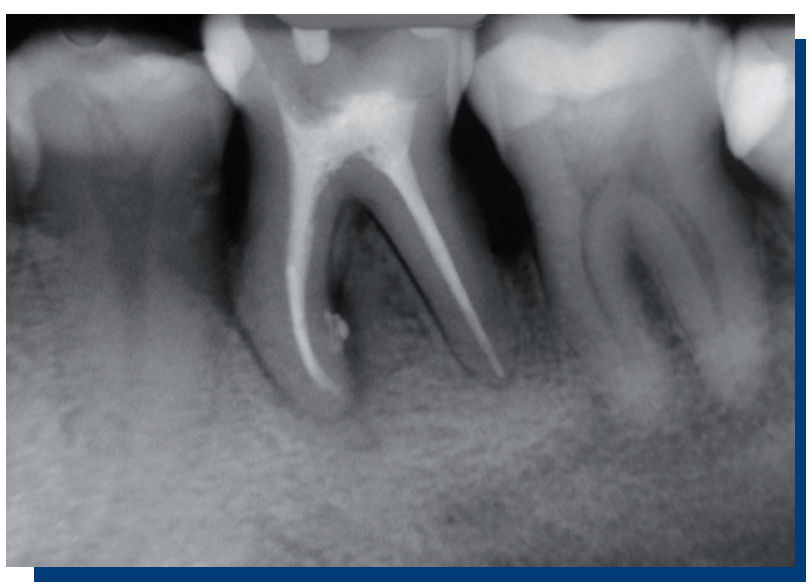

Figura 4. Control a los tres meses 


\section{DISCUSIÓN}

Ha sido demostrado que tanto las lesiones periodontales como pulpares pueden afectar el aparato de inserción, por tal motivo, antes de instituir una terapia se debe determinar si la lesión presente es de origen pulpar, periodontal o combinada ${ }^{(8)}$.

Las lesiones endoperiodontales verdaderas ocurren cuando existe una lesión periapical originada por una necrosis pulpar en un diente afectado periodontalmente, en que radiográficamente es posible observar el defecto infraóseo que se crea cuando ambas entidades se unen y emergen en algún lugar de la superficie radicular ${ }^{(9)}$. La reabsorción ósea marginal observada es similar a la que se encuentra en lesiones periodontales y el compromiso de la zona apical presenta un origen endodóntico en los cuales la pulpa no responde a los test de vitalidad. En estos casos se presenta clínicamente un saco periodontal profundo alrededor de la superficie del diente, lo que generalmente va asociado a una periodontitis generalizada ${ }^{(9)}$

Los procesos endoperiodontales, en general, requieren un tratamiento multidisciplinario para su evolución satisfactoria y es imprescindible el correcto diagnóstico para determinar la etiología de la lesión periradicular, basándose a su vez en signos clínicos y en la radiografía periapical(10).

El objetivo de la terapia es remover los factores etiológicos responsables de la destrucción tisular. Varias técnicas pueden ser empleadas para minimizar la destrucción tisular, para inducir la reparación de las estructuras de soporte, para prevenir la pérdida dentaria y mantener la integridad de la arcada dentaria(10).

Dependiendo de la etiología de la lesión, ésta puede responder solo con la terapia periodontal o endodóntica, pero cuando se está frente a lesiones combinadas, deben ser instituidas medidas más complejas, lo que reafirma la estrecha relación entre la endodoncia y la periodoncia. El tratamiento de elección es el procedimiento más simple con el que se pueda obtener el resultado terapéutico ideal(11). Para este fin, la terapia endodóntica asume una posición de gran importancia, con su aplicación se pueden satisfacer muchos de los objetivos de la terapia ${ }^{(8)}$.

El tratamiento de las lesiones endoperiodontales verdaderas, como el analizado en este caso, no difiere del efectuado cuando ambos cuadros ocurren por separado; el mismo debe ser instituido para obtener resultados óptimos en ambos problemas. La parte de la lesión que es producida por la infección del conducto radicular se resuelve después de un correcto tratamiento endodóntico; por otra parte la lesión producida por la infección de la placa bacteriana también mejora luego de realizar el tratamiento periodontal ${ }^{(12,13)}$.

Es importante comprender que clínicamente no es posible determinar hasta qué grado uno u otro de los trastornos ha afectado a los tejidos de sostén, por esto la estrategia de tratamiento deberá enfocarse primero a la infección pulpar, realizando de esta forma el tratamiento de conductos respectivo, debido a que algunas veces las lesiones periodontales mejoran después de un exitoso tratamiento endodóntico(12,13)

En el manejo de las lesiones endoperiodontales verdaderas, varios estudios apoyan realizar el tratamiento de conductos previo al tratamiento periodonta| ${ }^{(5,11,13)}$. Se considera esencial un periodo de observación de al menos un mes de evolución para permitir el potencial de regeneración tisular después del tratamiento endodóntico. Esta estrategia es ventajosa por tres razones; primero, el espacio endodóntico puede ser desinfectado mediante el tratamiento endodóntico, confinando la infección e irritantes bacterianos al defecto periodontal mediante el sellado del camino de comunicación entre la pulpa y el periodonto. La segunda razón consiste en que el raspado y alisado radicular puede interferir en el potencial de reinserción debido a que probablemente hayan fibras del ligamento periodontal insertadas e intactas que presentan potencial de regeneración del aparato de inserción tras el tratamiento de conductos $^{(13)}$. Y por último, si el tratamiento periodontal se realizara previo al endodóntico, la cicatrización periodontal puede comprometerse por los irritantes derivados del sistema de conductos radiculares ${ }^{(7)}$.

\section{CONCLUSIÓN}

La endodoncia y la periodoncia se consideran a menudo como entidades separadas, sin embargo tienen en común factores etiológicos y fisiopatológicos, los que clínicamente hacen que ambas especialidades estén estrechamente relacionadas, lo cual debe influenciar directamente en nuestro diagnóstico y tratamiento.

La comunicación entre los tejidos periodontales y pulpares puede producirse a través de los túbulos dentinarios, foramen apical, conductos laterales, fracturas radiculares o perforaciones. De tal manera, el diagnóstico de dichas afecciones es vital para llevar a cabo un acertado tratamiento y mejorar su pronóstico.

Es importante saber diagnosticar; para ello se deben utilizar todos los elementos clínicos o radiográficos disponibles en cada caso, de tal forma la endodoncia y la periodoncia son especialidades que deben trabajar en conjunto por las lesiones de ambos orígenes que se presentan en la práctica diaria.

En los casos en que se presente una lesión endoperiodontal verdadera, siempre habrá que abordar el caso desde el punto de vista endodóntico primero seguido de la terapia periodontal.

\section{REFERENCIAS BIBLIOGRÁFICAS}

1. Vakalis SV, Whitworth JM, Ellwood RP, Preshaw PM. A pilot study of treatment of periodontal-endodontic lesions. Int Dent J, 2005; 55(5): 313-318.

2. Rotstein I, Simon JHS. Diagnosis, prognosis and decision-making in the treatment of combined periodontal-endodontic lesions. Periodontol, 2000; 4: 165-203.

3. Sartori S, Silvestri M, Cattaneo V. Endoperiodontal lesion. A case report. J Clin Periodontol, 2002; 29(8): 781-783.

4. Chen SY, Wang HL, Glickman GN. The influence of endodontic treatment upon periodontal wound healing. Review. J Clin Periodontol, 1997; 24(7): 449-456.

5. Zehnder M, Gold SI, Hasselgren G. Pathologic interactions in pulpal and periodontal tissues. Review. J Clin Periodontol, 2002; 29(8): 663671

6. Rupf $S$ et al. Comparison of profiles of key periodontal pathogens in the periodontium and endodontium. Endod Dent Traumatol, 2000; 16: 269-275.

7. Chapple I, Lumley P. The periodontal-endodontic interface. Dental Update, 1999; 26(10): 331-341.
8. Kuang-Min Ch, Lin L. Diagnosis of an advance endodonticperiodontic lesion: Report of a case. Oral Surg Oral Med Oral Pathol, 1997; 84: 79-81.

9. Mandel Ely et al. Clinical diagnosis and treatment of endodontic and periodontal lesions. Quintessence International,1993; 24(2): 135-137. 10. Yuval Z, Avital K. Two approaches to the treatment of true combined periodontal-endodontal lesions. Journal of Endodontic, 1993; 19: 414416.

11. Solomon $\mathrm{Ch}$ et al. The endodontic-periodontal lesion: A rational approach to treatment. JADA, 1995; 126: 473-478.

12. Aqrabawi $\mathrm{J}$ et al. The healing potential of periodontal-endodontic lesions. International Dental Journal, 2004; 54: 166-170.

13. Paul BF, Hutter JW. The endodontic-periodontal continuum revisited: New insights into etiology, diagnosis and treatment. JADA 1997; 128: 1541-1548. 